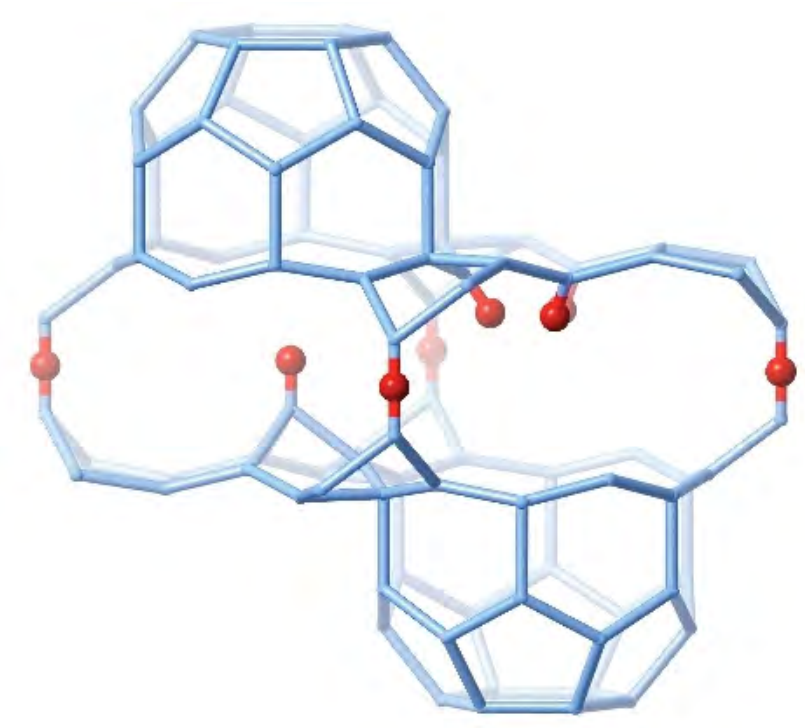

References:

[1] Smeets, S., Berkson, Z.J., Xie, D., Zones, S.I., Wan, W., Zou, X., Hsieh, M.-F., Chmelka, B.F., McCusker, L.B., \& Baerlocher, C. (2017) J. Am. Chem. Soc., 139, 16803-16812

[2] Zones, S. I., Burton, A. W. US7108843 B2, September 19, 2006.

Keywords: disorder, polycrystalline materials, zeolites
MS25-02

\section{Atomic scale 3D characterisation of hetero-nanosystems: a dose-efficient fusion of imaging and analytical techniques in scanning transmission electron microscopy}

Sandra Van Aert ${ }^{1}$,

1. EMAT - University of Antwerp, Antwerp, Belgium)

email: sandra.vanaert@uantwerpen.be

The properties of nanosystems are essentially determined by the positions of the atoms, their chemical nature and the bonding between them, but a fundamental understanding on the connection between the 3D structure and the properties at a local scale has been slow to emerge. Clearly, the rational design of nanomaterials with desired functional properties strongly depends on the availability of quantitative 3D characterisation techniques at the atomic scale. Aberration-corrected scanning transmission electron microscopy (STEM) has become a powerful technique for materials characterization of complex nanosystems. Recent progress in the development of quantitative methods allows us to extract structural and chemical information from experimental images in 2D as well as in 3D. In quantitative STEM, images are treated as datasets from which structure parameters are determined by comparison with image simulations or by using parameter estimation-based methods. In order to retrieve the $3 \mathrm{D}$ atomic structure, the use of scattering cross-sections to count atoms along the viewing direction has become a successful technique. Cross-sections define the total scattered intensity for each atomic column. Their high sensitivity in combination with a statistical analysis enables us to count atoms with single-atom sensitivity. These atom counts can be used to create an initial atomic model with which an energy minimization can be applied to obtain a relaxed $3 \mathrm{D}$ reconstruction of the nanoparticle. Recent progress enables us to extend atom-counting from homogeneous to heterogeneous materials. The introduction of several atomic elements into nanostructures can enhance properties such as stability, catalytic activity and electrical response. However, for mixed columns, all types of elements will contribute differently to the experimental scattering cross-sections, significantly complicating atom-counting as compared to monotype nanostructures. In order to extend atom-counting to heterogeneous materials, we introduced a physics-based atomic lensing model. This model is capable of predicting scattering cross-sections in terms of the 3D atomic arrangement creating new possibilities for unravelling the 3D composition. However, since scattering cross-sections depend on both sample composition and thickness, additional measurements are required to separate out those different contributions. In particular, energy dispersive X-ray spectroscopy combined with STEM imaging enables us to count the number of atoms in hetero-nanosystems without the need for prior knowledge. This multimodal approach can even be used to unravel small atomic number differences. Finally, new quantitative STEM strategies using a so-called fast pixelated detector will be shown enabling us to unravel thickness, composition, and even the oxidation state and 3D ordering of atoms.

Keywords: scanning transmission electron microscopy, quantitative structure characterisation, hetero-nanosystems 